\title{
CS based confocal microwave imaging algorithm for breast cancer detection
}

\author{
Y.P. Sun ${ }^{\mathrm{a}, \mathrm{b}, *}$, S. Zhang ${ }^{\mathrm{a}}$, Z. Cui ${ }^{\mathrm{b}}$ and L.L. Qu ${ }^{\mathrm{b}}$ \\ ${ }^{a}$ College of Information Science and Engineering, Northeastern University, Liaoning, China \\ ${ }^{\mathrm{b}}$ Department of Electronic Information Engineering, Shenyang Aerospace University, Liaoning, China
}

\begin{abstract}
Based on compressive sensing (CS) technology, a high resolution confocal microwave imaging algorithm is proposed for breast cancer detection. With the exploitation of the spatial sparsity of the target space, the proposed image reconstruction problem is cast within the framework of CS and solved by the sparse constraint optimization. The effectiveness and validity of the proposed CS imaging method is verified by the full wave synthetic data from numerical breast phantom using finitedifference time-domain (FDTD) method. The imaging results have shown that the proposed imaging scheme can improve the imaging quality while significantly reducing the amount of data measurements and collection time when compared to the traditional delay-and-sum imaging algorithm.
\end{abstract}

Keywords: Microwave imaging, breast cancer detection, compressive sensing (CS), FDTD method

\section{Introduction}

Breast cancer is one of the most terrible disease effecting women all over the world [1]. Due to the high incidence rate of its occurring, the efficient diagnose technology for breast cancer detection is in urgent need. The earlier breast cancer is found, the easier it is to treat with. Currently, X-ray mammography is the most effective technique for breast cancer detection [2]. However, it suffers from relatively high missed and false-detection rates and has the underlying risk of the destructiveness to cells and nerves of patients. Some other available screening techniques such as MRI and ultrasound are either too costly or less effective. The shortcomings of these techniques motivate the development of the alternative technique for breast cancer detection.

The significant contrast in the dielectric properties at microwave frequencies of normal and malignant breast tissue has provided an opportunity for the employment of the microwave breast imaging (MBI) [3, 4]. Since MBI is non-ionizing and noninvasive in practice, it has received the increasing attention in the area of the breast cancer detection. The main challenge of MBI for breast cancer detection is to develop a robust imaging algorithm that can provide the high quality image with the high resolution and good suppression of artifacts [5].

Over the past years, a wide variety of MBI approaches have been proposed in the reported literature. These algorithms can be generally divided into two main categories. The first category is microwave

\footnotetext{
${ }^{*}$ Corresponding author: Y.P. Sun, College of Information Science and Engineering, Northeastern University, No. 11 Wenhua Road 110819, Liaoning, China. E-mail: polopolo88@126.com.
} 


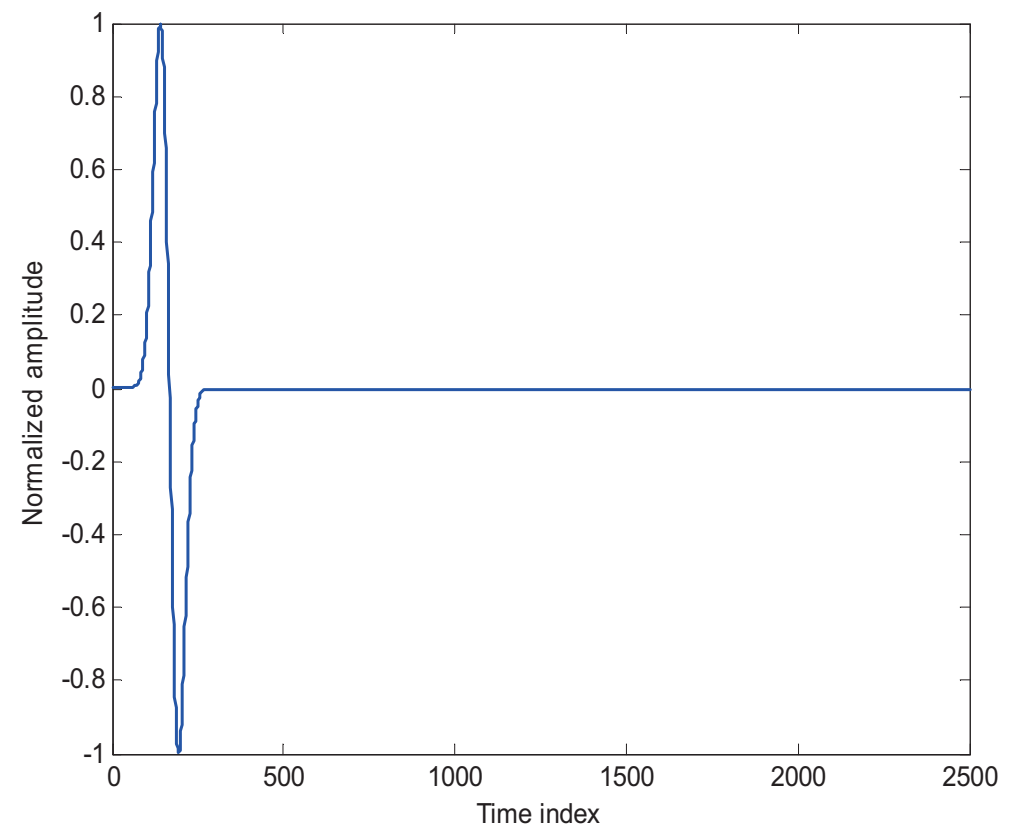

Fig. 1. Waveform of transmitted impulse signal.

tomographic method which involves the solution of an ill-conditioned nonlinear inverse scattering problem [6]. The microwave tomography can recover the electrical properties profile of breast from the measured data. The microwave tomographic algorithm is usually vulnerable to the noise and computationally intensive. The second category is the ultra-wideband (UWB) microwave confocal imaging method which uses synthetic or physical apertures to infer the locations of significant microwave scatters such as malignant tumors [7]. Within the framework of UWB microwave imaging, the delay-and-sum (DAS) beamforming algorithm and its variation have been developed for breast cancer detection. The images provided by DAS-kind algorithms may suffer from low resolution and high sidelobe levels.

In this paper, a new approach of compressive sensing (CS) [8] based MBI algorithm for breast cancer detection is proposed. By exploiting the spatial sparsity of the imaging space, the MBI reconstruction problem is cast within the framework of CS and solved by the sparse constraint optimization. The effectiveness and validity of the proposed CS MBI method is verified by the full wave synthetic data from numerical breast phantom using finite-difference time-domain (FDTD) method [9]. The imaging results have shown that the proposed imaging scheme can improve the imaging quality while significantly reducing the amount of data measurements and collect time.

The remainder of this paper is organized as follows. The adopted breast phantom model is described In Section 2. In Section 3, the proposed CS confocal imaging algorithm is given in detail explanation. In Section 4, the result and performance of CS based reconstruction imaging algorithm are presented and analyzed. Finally, concluding remarks are summarized in Section 5.

\section{FDTD breast model for data acquisition}

In conventional microwaves imaging systems [10], the bistatic pattern is to make the transmitting and receiving antennas move together with a fixed offset over the investigation domain. The correspond- 


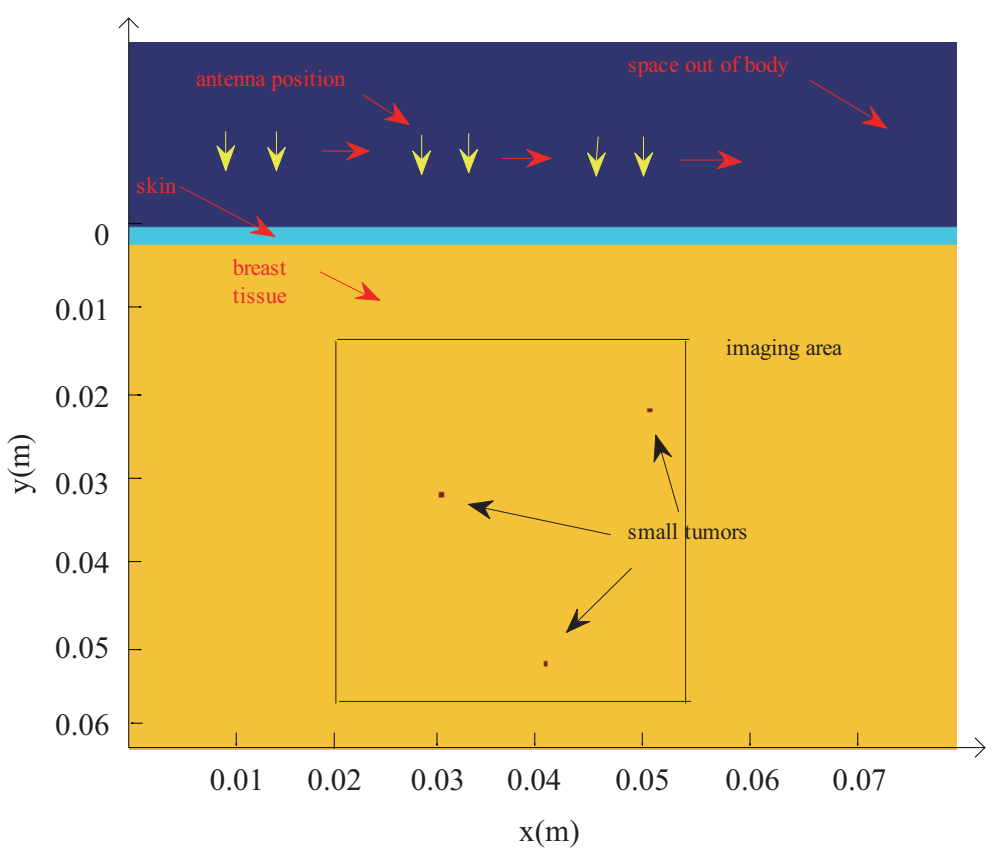

Fig. 2. 2-D FDTD Breast Phantom model.

ing movement of antennas provides larger synthetic aperture than a single antenna aperture, so high resolution imaging results in the crossrange will be obtained [11].

A bistatic antenna array consists of two elements: a transmitting antenna and a receiving antenna, set them at a short distance from the surface of the breast phantom and move them together with a fixed distance when the detecting procedure is in process. The transmitting antenna activates impulse signals to the breast phantom continuously, and the returned signals are acquired by the receiving antenna.

The expression of the transmitted impulse signal is the second order derivative of Gaussian function [12], the transmitted waveform is depicted on Fig. 1 and it can be expressed by Eq. (1).

$$
s(t)=-2 \xi \sqrt{e^{-\frac{1}{2 \xi}}} e^{-\xi(t-\alpha)^{2}}(t-\alpha)
$$

where $f_{c}$ is the center frequency, $\alpha=1 / f_{c}$ and $\xi=2 \pi^{2} f_{c}^{2}$.

To simulate a realistic breast cancer detection scenario, a 2-D numerical breast phantom model is constructed to generate the full wave synthetic data by using FDTD method [13]. The 2-D numerical breast phantom is illustrated in Fig. 2. The 2-D breast phantom model includes a 2-mm-thick skin layer with a relative permittivity $\left(\varepsilon_{1}=36\right)$. Three square malignant tumors $\left(\varepsilon_{2}=50\right)$ with side equal to $0.5 \mathrm{~mm}$ are embedded in the normal breast tissue $\left(\varepsilon_{3}=9\right)$. The synthetic aperture consists of 41 antenna locations. Both of the transmitter and receiver move in the step of $0.12 \mathrm{~cm}$ to implement $4.8 \mathrm{~cm}$ synthetic aperture. The grid size in the 2-D FDTD model is set to $\Delta x=\Delta y=0.0005 \mathrm{~m}$ and the time step is $1.18 \mathrm{ps}$. The 2-D FDTD problem space is terminated with perfectly matched layer (PML) boundaries [14].

The time domain response is collected at each receiver location and the measurement data generated by FDTD is shown in Fig. 3, which consists of $41 \times 2120$ space-time measurements. It is observed that the location of three tumors can not be easily obtained in Fig. 3. It is necessary to employ the appropriate the imaging algorithm to process the measurement data to obtain the location of three tumors. 


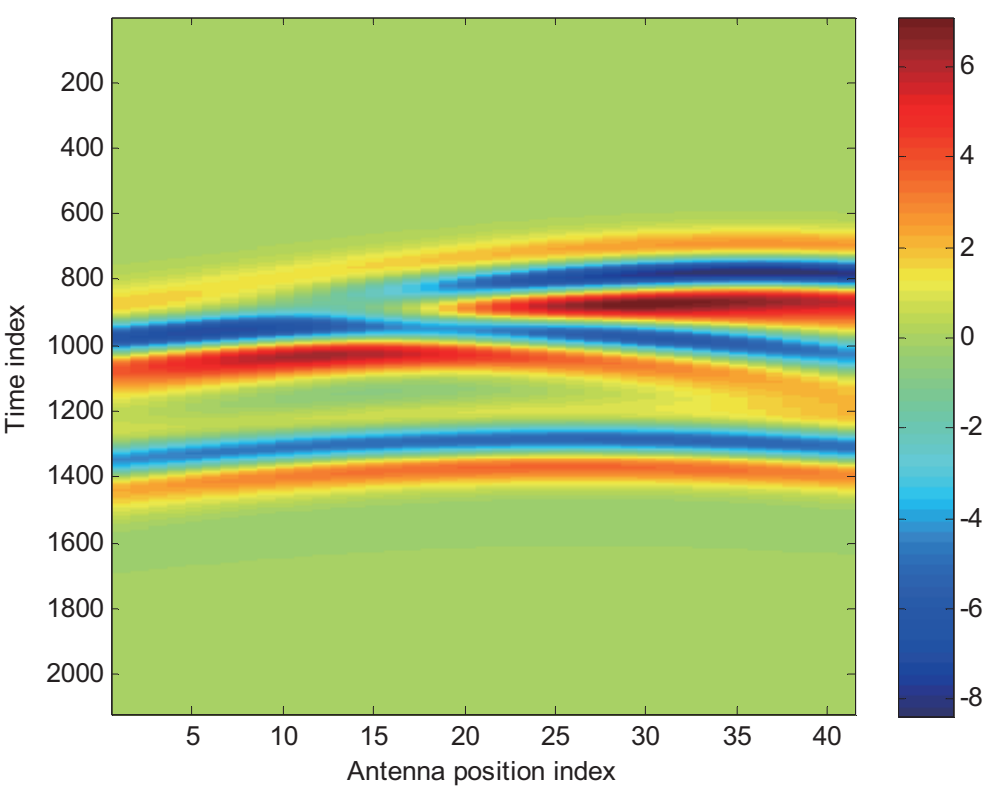

Fig. 3. The measured time domain data.

\section{CS reconstruction imaging}

\subsection{The received signal model}

Assume that there are $P$ tumor targets in the breast phantom model, the returned signal at the $m$ th antenna location is given in Eq. (2).

$$
\beta_{m}(t)=\sum_{p=0}^{P-1} \sigma_{p} s\left(t-\tau_{p m}\right)
$$

where $\sigma_{p}$ represents the complex reflectivity of the $p$ th tumor target, $\tau_{p m}$ is the round-trip propagation time delay of the $p$ th tumor target for the $m$ th antenna location. $\tau_{p m}$ is determined by the propagation path of the $p$ th tumor target corresponding to the $m$ th antenna location.

The propagation path for the tumor target in the breast phantom model is demonstrated in Fig. 4. The accurate refraction points corresponding to the interfaces of each two different mediums can be obtained by solving via Snell's law [15]. After the refraction points are obtained, the round-trip propagation timedelay in each medium can be calculated by dividing the round-trip propagation path by the corresponding speed. Finally the total round-trip propagation time-delay can be obtained by summing the round-trip propagation time delay of each medium.

\subsection{Sparsity-based data acquisition and reconstruction}

Consider that the imaging region of interest is divided into a finite number of pixels $N_{x} \times N_{y}$ in crossrange and downrange, and the tumor targets occupy no more than $P\left(<<N_{x} \times N_{y}\right)$ pixels. Let $r(k, l), k=0, \cdots, N_{x}-1, l=0, \cdots, N_{y}-1$ be a weighted indicator function, which takes the value $\sigma_{p}$ if the $p$ th target exists at the $(k, l)$ th pixel, otherwise, it is zero. If $r(k, l)$ is lexicographically 


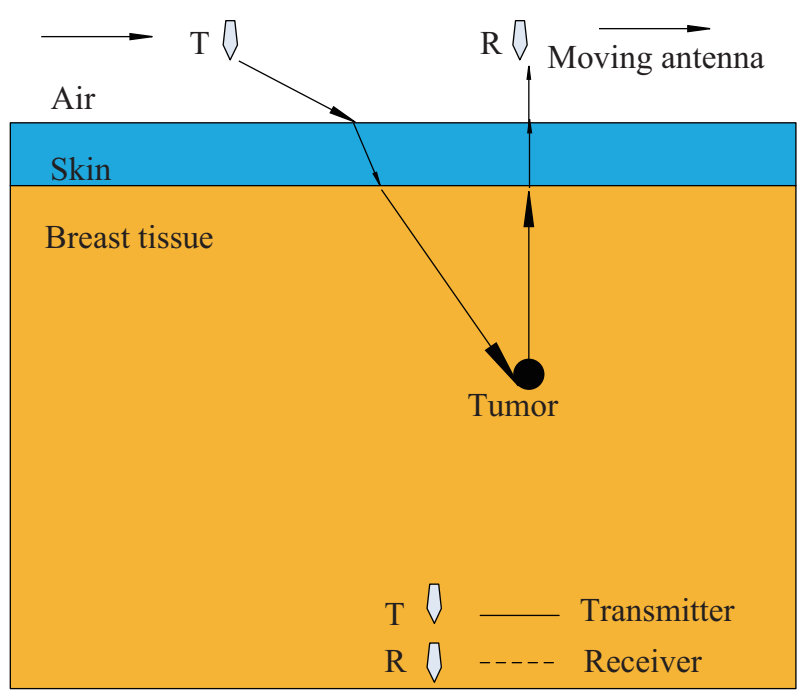

Fig. 4. Detailed propagation path.

ordered into a column vector $\mathbf{r}$ of length $T=N_{x} N_{y}$, the received signal of Eq. (2) can be represented in matrix-vector form as is shown in Eq. (3).

$$
\boldsymbol{\beta}_{m}=\boldsymbol{\Psi}_{m} \mathbf{r}
$$

where $\boldsymbol{\beta}_{m}=\left[\beta_{m}\left(t_{0}\right), \beta_{m}\left(t_{1}\right), \cdots, \beta_{m}\left(t_{N-1}\right)\right], t_{0}$ is the initial measurement time, $t_{N-1}=t_{0}+(N-$ 1) $\Delta t, \Delta t$ is the sampling time and $N$ is the total number of time samples. The $j$ th column of the $N \times T$ matrix $\Psi_{m}$ can be expressed as Eq. (4).

$$
\left[\mathbf{\Psi}_{m}\right]_{j}=\frac{s\left(t-\tau_{j m}\right)}{\left\|s\left(t-\tau_{j m}\right)\right\|_{2}}, t=t_{0,} \cdots, t_{N-1}
$$

For CS based MBI scheme, a reduced set of measurements $\mathbf{y}_{m}$ is measured instead of directly measuring the $\mathrm{N}$-dimensional signal $\boldsymbol{\beta}_{m}$ by introducing the measurement matrix Eq. (5).

$$
\mathbf{y}_{m}=\boldsymbol{\Phi}_{m} \boldsymbol{\beta}_{m}=\boldsymbol{\Phi}_{m} \boldsymbol{\Psi}_{m} \mathbf{r}+\mathbf{n}_{m}
$$

where $\boldsymbol{\Phi}_{m}$ is a $J \times N$ measurement matrix constructed by randomly selecting $J$ rows of an $N \times N$ identity matrix. Without loss of generality, an additive noise vector $\mathbf{n}_{m}$ is added in Eq. (5). By employing this data acquisition strategy, the $\mathrm{N}$-dimensional signal $\boldsymbol{\beta}_{m}$ is condensed into a J-dimensional signal $\mathbf{y}_{m}$.

Assume that there are $M$ antenna locations, superpose the matrix equation in Eq. (5) for all $M$ antenna locations to form a composite matrix in Eq. (6).

$$
\mathbf{y}=\mathbf{\Phi} \Psi \mathbf{r}=\mathbf{D r}+\mathbf{n}
$$

where $\mathbf{y}=\left[\mathbf{y}_{1}^{T}, \mathbf{y}_{2}^{T}, \cdots, \mathbf{y}_{M}^{T}\right]^{T}, \mathbf{\Phi}=\operatorname{diag}\left[\boldsymbol{\Phi}_{1}, \boldsymbol{\Phi}_{2}, \cdots, \boldsymbol{\Phi}_{M}\right], \mathbf{\Psi}=\left[\boldsymbol{\Psi}_{1}^{T}, \boldsymbol{\Psi}_{2}^{T}, \cdots, \boldsymbol{\Psi}_{M}^{T}\right]^{T}, \mathbf{n}=$ $\left[\mathbf{n}_{1}^{T}, \mathbf{n}_{2}^{T}, \cdots, \mathbf{n}_{M}^{T}\right]^{T}$ and $\mathbf{D}=\boldsymbol{\Phi} \boldsymbol{\Psi}$. The sparse signal $\mathbf{r}$ can be recovered from $\mathbf{y}$ by solving the following $l_{1}$-norm optimization problem in Eq. (7).

$$
\min _{\mathbf{r}}\|\mathbf{r}\|_{l_{1}} \text { s.t. } \quad \mathbf{y} \approx \mathbf{D r}
$$




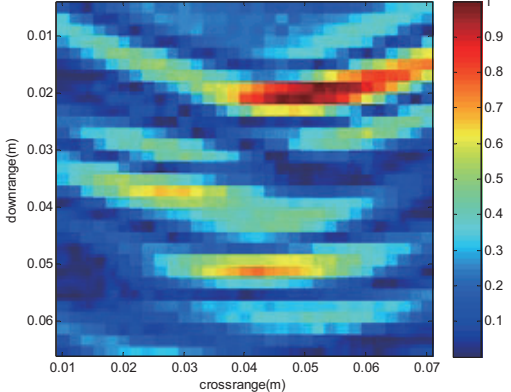

(a)

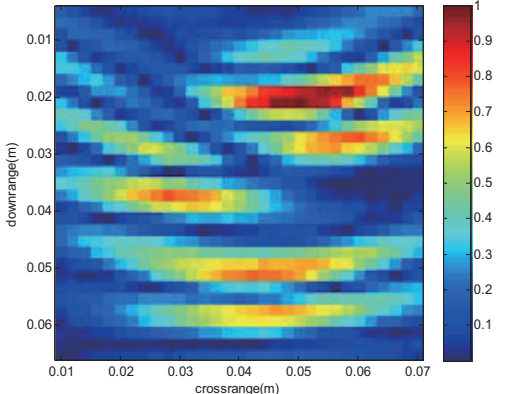

(b)

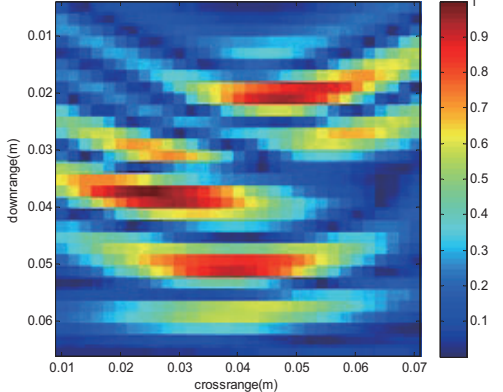

(c)

Fig. 5. Image of three tumor targets using DAS algorithm; (a) DAS with $12.5 \%$ of full data; (b) DAS with $25 \%$ of full data; (c) DAS with $50 \%$ of full data.

The problem in Eq. (7) can be solved by using basis pursuit, greedy pursuit, or combinatorial algorithms. In this paper, orthogonal matching pursuit (OMP) [16] is taken into use, which is known to provide a fast and easy approach to implement solution. It is noted that the number of iterations of OMP is usually associated with the sparse level of the reconstructed image. In practice, this piece of information is often unavailable, and the stopping condition is heuristic. The cross-validation technique can be used to determine the stopping condition for OMP reconstruction algorithm.

\section{Simulation results}

In order to demonstrate the performance of the proposed CS based imaging algorithm for breast cancer detection, several numerical examples are presented in this section.

The image area starts from the place which is $3 \mathrm{~mm}$ from the top surface of skin layer and its scope is $6 \mathrm{~cm}$ and $7 \mathrm{~cm}$ in crossrange and downrange directions respectively. The synthetic array consists of $M=41$ transmitter-receiver pairs equally spaced at $0.0015 \mathrm{~m}$ intervals. The transmitter and receiver has a fixed offset distance of $0.0005 \mathrm{~m}$. The impulse signal used as the antenna excitation is a second order derivative Gaussian pulse with a center frequency of $2.5 \mathrm{GHz}$. In order to ensure the enough propagation time for the round-trip of signals, a proper $2.5 \mathrm{~ns}$ time-window is chosen for sampling in the time domain. Additive complex white Gaussian noise is added to the measurements with a signal-to-noise ratio (SNR) of $0 \mathrm{~dB}$, the SNR is defined as the ratio of the total signal power to the noise power in the time domain.

In fact, the recorded signals include early-time and late-time contents. The early-time contents corresponds for the reflections from the skin and antenna reverberations, which lie on the early part of the time domain with high amplitudes, whereas the late-time contents contain the information of the tumor response and clutter. Since the early-time contents appear earlier than the tumor responses in the time domain, a time-gating technique could be used here to eliminate them. Make good choice of time gate ensures that the image could be reconstructed with good robustness.

A reduced set of measurements is taken into CS-based reconstruction, it is an randomly sampled matrix of original received signals with $M=41$ in columns and $N=212$ in rows. The reflectivities of the tumor targets are assumed to be the same and constant, independent of frequency and aspect angles [17].

Figure 5 displays the images reconstructed by using the traditional DAS algorithm with different compression ratios. The compression ratio is defined as the ratio between the randomly selected data and 
Table 1

Detailed performance of two reconstruction algorithms

\begin{tabular}{lccc}
\hline Imaging methods & Measurement ratio & Intensity of tumor & Computational time \\
\hline DAS algorithm & $12.5 \%$ & $(0.423,0.551,0.557)$ & $9.364 \mathrm{~s}$ \\
& $25 \%$ & $(0.568,0.532,0.743)$ & $9.651 \mathrm{~s}$ \\
& $50 \%$ & $(0.747,0.778,0.767)$ & $10.364 \mathrm{~s}$ \\
CS-based algorithm & $12.5 \%$ & $(0.453,0.673,0.961)$ & $0.832 \mathrm{~s}$ \\
& $25 \%$ & $(0.656,0.871,0.992)$ & $1.024 \mathrm{~s}$ \\
& $50 \%$ & $(0.993,0.992,0.999)$ & $1.551 \mathrm{~s}$ \\
\hline
\end{tabular}

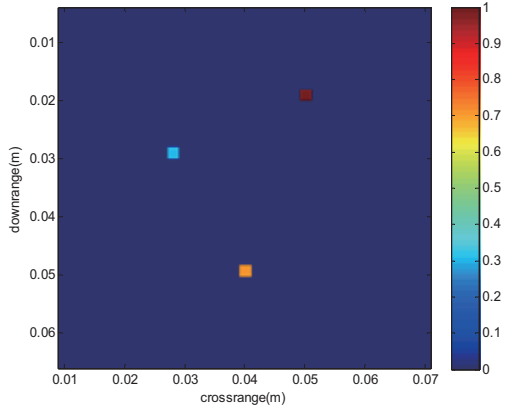

(a)

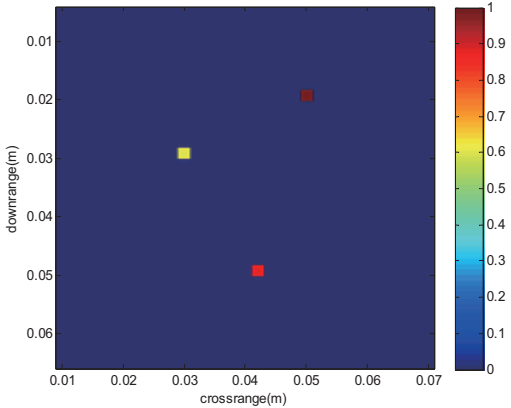

(b)

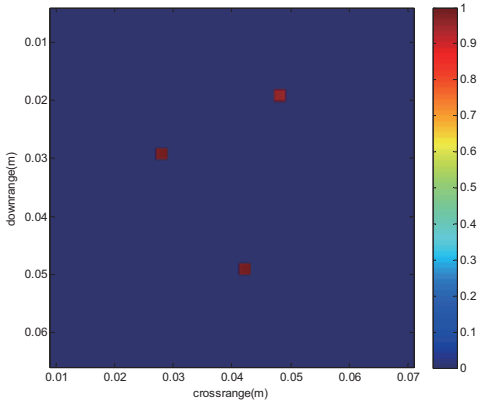

(c)

Fig. 6. Image of three tumor targets using CS based algorithm. (a) CS with a compression ratio of $12.5 \%$; (b) CS with a compression ratio of $25 \%$; (c) CS with a compression ratio of $50 \%$.

the full measurement data at each antenna location. Figure 6 shows the reconstructed images obtained by the proposed CS-based imaging algorithm with different compression ratios.

To make the observation more intuitively, each of the reconstructed images is plotted with the maximum intensity value normalized to its maximum and the authenticity of reconstructed targets parameters can be expressed through pixel values directly. It is obvious that the CS-based reconstruction algorithm performs much better than the DAS algorithm in terms of reducing the measurement data and suppressing the clutter responses. The high clutter responses in the reconstructed images obtained by the DAS algorithm bring the significant mess to the reconstructed image area. It is hard to locate the accurate position of tumor targets in the DAS reconstructed images. However, with the same simulation layouts, as is shown in Fig. 6, although the measurement data is significantly reduced, the CS-based reconstruction algorithm can still reconstruct the tumor targets with higher resolution and less sidelobe level.

Due to the high efficiency of OMP algorithm, the CS-based method can provide much better imaging results with less computational time. The detailed computational time of two different algorithms is given in Table 1. For each image, the intensity values in the brackets are respectively corresponding to the tumor targets from left to right in the crossrange.

In order to have the quantitative analysis on the result of the experiments, two metrics are introduced in Eqs (8) and (9), respectively. The normalized mean of squared error (NMSE) is defined to measure the reconstruction error:

$$
N M S E=\left\|z-z^{\prime}\right\|_{2} /\|z\|_{2}
$$

where $z^{\prime}$ and $z$ are the reconstructed and true signals, respectively. The image quality is measured using the target-to clutter ratio (TCR) [18]:

$$
T C R=10 \log 10\left(P / P^{\prime}\right)
$$




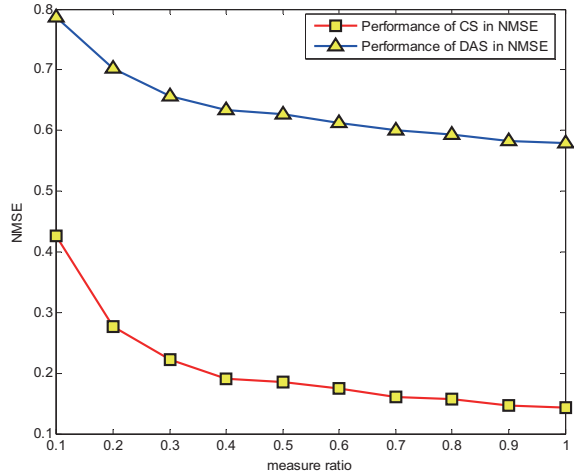

(a)

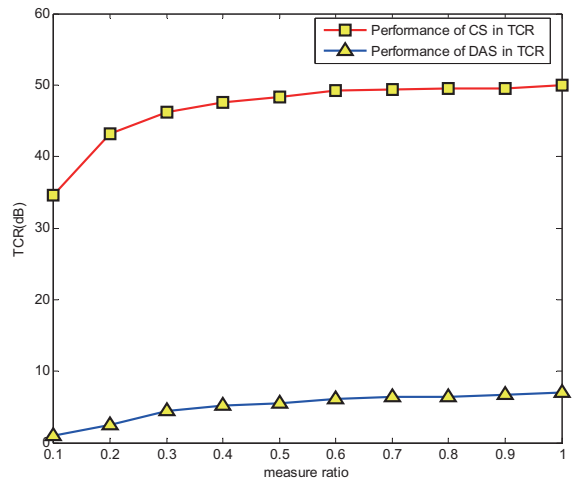

(b)

Fig. 7. Quantitative comparison of the reconstructed performance for two imaging methods. (a) Measurements of Average NMSE; (b) Measurements of Average TCR.

where $P$ and $P^{\prime}$ are the average power of the target and clutter regions, respectively. For each quantitative analysis measurements, the experiment is operated with 20 times of Monte-Carlo simulation, and the average NMSE and TCR were recorded. The performance of two different algorithms is given in Fig. 7 .

It is well observed that the image reconstructed by the CS based algorithm performs far better than traditional DAS algorithm with significantly lower reconstruction error, and the targets are well localized with high intensity values.

\section{Conclusion}

A novel CS based confocal microwave imaging approach for breast cancer detection have been presented in the paper. The proposed CS based imaging scheme which exploits the sparsity of the target space can significantly reduce the data acquisition time by decreasing the total number of measurements. The OMP algorithm is used to reconstruct the image from a small number of random space-time measurements. The experimental results from full wave synthetic data have shown that the proposed imaging scheme can improve the reconstruction quality and significantly reduce the amount of data measurements when compared to the traditional DAS imaging algorithm.

\section{Acknowledgments}

This work was supported in part by the National Natural Science Foundation of China under Grant 61302172, by the Natural Science Foundation of Liaoning Province under Grant 2014024002, by the Doctoral Startup Foundation of Liaoning Province under Grant 20121035 and 20131085, and by the Scientific Research Project of the Department of Education of Liaoning Province under Grant L2013069.

\section{References}

[1] J. Bourqui, C. Curtis, D. Mew, B. Docktor and C. Romano, Microwave breast imaging with a mono static radar-based system: A study of application to patients. IEEE Transactions on Microwave Theory and Techniques 61, 2119 (2013). 
[2] M. Patlak, S.J. Nass, I.C. Henderson and J.C. Lashof, Mammography and Beyond: Developing Techniques for the Early Detection of Breast Cancer, Washington, DC: Inst. of Med, National Academy Press (2000).

[3] M. Klemm, J.A. Leendertz and D. Gibbins, Microwave radar-based differential breast cancer imaging: Imaging in homogeneous breast phantoms and low contrast scenarios. IEEE Transactions on Antennas and Propagation 58, 2337 (2010).

[4] B. Makladet, C. Curtis and E.C. Fear, Neighborhood-based algorithm to facilitate the reduction of skin reflections in radar-based microwave imaging. Progress in Electromagnetics Research B 39, 115 (2012).

[5] J. Bourqui, J.M. Sill and E.C. Fear, A prototype system for measuring microwave frequency reflections from the breast. International Journal of Biomedical Imaging 2012, 851234 (2012).

[6] T.M. Grzegorczyk, P.M. Meaney and P.A. Kaufman, Fast 3-D tomographic microwave imaging for breast cancer detection. IEEE Transactions on Medical Imaging 31, 1584 (2012).

[7] J. Bourquiet, J. Garrett and F. Elise, Measurement and analysis of microwave frequency signals transmitted through the breast. International Journal of Biomedical Imaging 2012, 562563 (2012).

[8] D. Donoho, Compressed sensing. IEEE Transactions on Information Theory 52, 1289 (2006).

[9] M.A. Eleiwa and A.Z. Elsherbeni, Accurate FDTD simulation of biological tissues for bio-electromagnetic applications. Proc. IEEE Southeast Conference (2001), pp. 174-178.

[10] E.C. Fear, X. Li, S.C. Hagness and M.A. Stuchly, Confocal microwave imaging for breast cancer detection: Localization of tumors in three dimensions. IEEE Transactions on Bio-medical Engineering 49, 812 (2002).

[11] J. Bourquiet, M. Okoniewski and E.C. Fear, Balanced antipodal Vivaldi antenna with dielectric director for near-field microwave imaging. IEEE Transactions on Antennas and Propagation 58, 2318 (2010).

[12] M. Lazebnik, L. McCartney, D. Popovic and C.B. Watkins, A large-scale study of the ultra wideband microwave dielectric properties of normal breast tissue obtained from reduction surgeries. Physics in Medicine and Biology 52, 2637 (2007).

[13] P. Kosmas and C.M. Rappaport, FDTD-based time reversal for microwave breast cancer detection localization in three dimensions. IEEE Transactions on Microwave Theory and Techniques 54, 1921 (2006).

[14] J. Ernst, H. Maurer, A. Green and K. Holliger, Full-waveform inversion of cross hole radar data based on 2-D finitedifference time-domain solutions of Maxwell's equations. IEEE Transactions on Geoscience and Remote Sensing 45, 2807 (2007).

[15] M. Leigsnering, M.G. Amin, F. Ahmad and A.M. Zoubir, Multipath exploitation and suppression for SAR imaging of building interiors. IEEE Signal Processing Magazine 31, 111 (2014).

[16] D. Needell and R. Vershynin, Uniform uncertainty principle and signal recovery via regularized orthogonal matching pursuit. Foundations of Computational Mathematics 3, 317 (2009).

[17] M. Klemm, Contrast-enhanced breast cancer detection using dynamic microwave imaging. Antennas and Propagation Society International Symposium (APSURSI). IEEE (2012), pp. 1-2.

[18] C.H. Seng, A. Bouzerdoum, M.G. Amin and S.L. Phung, Two-stage fuzzy fusion with applications to through-the-wall radar imaging. IEEE Geoscience and Remote Sensing Letters 10, 687 (2012). 